\title{
Soziale Teilhabe durch geförderte Beschäftigung? Das Beispiel des Beschäftigungszuschusses
}

Der 2007 eingeführte Beschäftigungszuschuss (\$16e) ergänzt das Zweite Sozialgesetzbuch (SGB II) um eine Maßnahme für schwer vermittelbare Langzeiterwerbslose.

Während die Beschäftigungsförderung des SGB II vorrangig auf die Eingliederung der Leistungsempfänger ${ }^{\bullet}$ in den ersten Arbeitsmarkt zielt, setzt dieses auch als „JobPerspektive“ bekannte Instrument deutlich andere Akzente. Mit der staatlichen Förderung von längerfristigen, eventuell sogar dauerhaften Arbeitsverhältnissen geht es darum, mit Langzeiterwerbslosigkeit verbundene Ausgrenzungsrisiken zu überwinden und die Teilhabechancen schwer vermittelbarer Hilfebezieher nachhaltig zu stärken. Der folgende Beitrag diskutiert auf Grundlage qualitativer Befragungsdaten, ob und unter welchen Voraussetzungen diese Zielsetzung zu realisieren ist. ${ }^{\circledR}$

ANDREAS HIRSELAND, PHILIPP RAMOS LOBATO, TOBIAS RITTER

\section{Einleitung}

Öffentlich geförderte Beschäftigung gehört zu den klassischen Instrumenten aktiver Arbeitsmarktpolitik in Deutschland. Hierzu zählen die mit dem Arbeitsförderungsgesetz (AFG) eingeführten Arbeitsbeschaffungsmaßnahmen (ABM), aber auch die zunächst im Rahmen des Bundessozialhilfegesetzes seit 2005 in der neugeschaffenen „Grundsicherung für Arbeitsuchende“ eingesetzten Arbeitsgelegenheiten (AGH). Mit dem Beschäftigungszuschuss nach $\$ 16$ e SGB II (bis Januar $2010 \$ 16 a$ ) kam 2007 eine weitere Form öffentlich geförderter Beschäftigung hinzu. Der Einsatz dieser arbeitsmarktpolitischen Instrumente ist mit einer Reihe unterschiedlicher Zielsetzungen verbunden, die von der öffentlichen Infrastrukturpflege über Marktersatz und Zielgruppeninklusion bis hin zur Verbesserung der Beschäftigungsfähigkeit Erwerbsloser und deren Arbeitsmarktintegration reichen (vgl. Bellmann et al. 2006; Matysik et al. 2011, S. 10f.). Veränderungen auf dem Arbeitsmarkt sowie darauf bezogene arbeitsmarktpolitische Reformen führten in den vergangenen Jahrzehnten wiederholt zu einer Neugewichtung dieser Zieldimensionen. So wurde mit dem Übergang zur aktivierenden Arbeitsmarktpolitik die Verbesserung der Beschäftigungsfähigkeit zur zentralen Zieldimension erhoben. Im Kontext des SGB II dient der Einsatz öffentlich geförderter Beschäftigung zugleich der Überprüfung der Arbeitsbereitschaft und verleiht im Grundsatz „Fördern und Fordern“ dem „Workfare“Gedanken Ausdruck (vgl. Lessenich 2008).

Mit dem 2007 eingeführten Beschäftigungszuschuss für die Leistungsempfänger im SGB II mit besonders geringen Eingliederungschancen auf dem ersten Arbeitsmarkt - deren Anzahl je nach Definition im Dezember 2005 zwischen rund 130.000 und einer halben Million betrug (vgl. Koch/ Kupka 2007, S. 14) - gewinnt eine stärker sozialpolitisch motivierte Zielsetzung (wieder) an Bedeutung. Durch die

Im vorliegenden Beitrag wird aus Gründen der besseren
Lesbarkeit i.d.R. auch bei nicht geschlechtsneutralen Bezeichnungen die männliche Form verwendet.

2 Die vorgestellten Untersuchungsergebnisse sind im Rahmen der Begleitforschung zur Beschäftigungsförderung nach § 16e SGB II entstanden, die im Auftrag des Bundesministeriums für Arbeit und Soziales von einem Forschungskonsortium - bestehend aus dem Institut für Sozialforschung und Gesellschaftspolitik GmbH (ISG), Köln, dem Rheinisch-Westfälischen Institut für Wirtschaftsforschung e.V. (RWI), Essen, und dem Institut für Arbeitsmarktund Berufsforschung (IAB), Nürnberg - durchgeführt wurde. Zu weiteren Ergebnissen der Untersuchung vgl. Koch et al. 2010. Teile der Erhebung der qualitativen Studie zu den Teilhabeeffekten wurden an das Institut für sozialwissenschaftliche Forschung e.V. (ISF) München vergeben. Wir danken den beiden anonymen Gutachtern der WSI-Mitteilungen für ihre konstruktive Kritik zu einer Vorfassung dieses Beitrages. 
Integration dieser SGB-II-Leistungsempfänger in geförderte Erwerbsarbeit sollen die mit Langzeiterwerbslosigkeit verbundenen Ausgrenzungsrisiken und Teilhabedefizite überwunden werden. Damit kann dieses - auch nach der jüngsten Instrumentenreform in modifizierter Form beibehaltene - Instrument als Beispiel einer Stärkung der sozialpolitischen Komponente von Arbeitsmarktpolitik gelten, die insbesondere auf jene Gruppe von Langzeiterwerbslosen zielt, für die selbst in Zeiten einer verbesserten Arbeitsmarktlage kaum realistische Vermittlungschancen in reguläre Erwerbsarbeit bestehen.

Ob und inwieweit geförderte Beschäftigung diesem Anspruch gerecht wird, bemisst sich auch an ihrem Beitrag zur Stärkung sozialer Teilhabechancen von Langzeiterwerbslosen. Der vorliegende Beitrag möchte unter Rückgriff auf Ergebnisse einer qualitativen Befragung von nach $₫ 16 \mathrm{e}$ SGB II geförderten Personen zeigen, dass die Realisierung sozialer Teilhabechancen durch Integration in geförderte Erwerbsarbeit immer auch abhängig ist von unterschiedlichen subjektiven Aneignungsformen und Bewältigungsmustern einer Lebenssituation, die sich für die Betroffenen mit der Beschäftigungsaufnahme verändert hat. Der Ergebnispräsentation (Abschnitt 5) sind Informationen zu geförderter Beschäftigung im SGB II (2), zur Ausgestaltung des Beschäftigungszuschusses (3) sowie zur sozialwissenschaftlichen Verortung der intendierten Teilhabeeffekte geförderter Beschäftigung (4) vorangestellt. Der Beitrag schließt mit Überlegungen zu einigen strukturellen Bedingungen von Teilhabechancen im Zusammenhang mit geförderter Beschäftigung (6).

\section{2. Öffentlich geförderte Beschäftigung im SGB II}

Die mit dem AFG verbundene Zielsetzung geförderter Beschäftigung, bestehende Nachfragedefizite auf dem Arbeitsmarkt notfalls auch längerfristig auszugleichen, hat in den vergangenen beiden Jahrzehnten einen „deutlichen Akzeptanzverlust erlitten“" (Matysik et al. 2011, S. 8). Diese Marktersatzfunktion stand bis in die 1990er Jahre hinein im Zentrum der Beschäftigungsförderung, was angesichts ausgeprägter Marktungleichgewichte bereits in den 1980er Jahren und weiter verschärft durch die Folgen der Wiedervereinigung zu steigenden Förderquoten auch von ABM führte. Zudem waren unter dem Aspekt der Strukturwirksamkeit vor allem in den neuen Bundesländern infrastrukturelle Zielsetzungen bedeutsam (vgl. ebd., S. 12). Mit dem Mitte der 90er Jahre eingeleiteten Übergang zur stärker $a k$ tivierenden Arbeitsmarktpolitik war auch eine Neujustierung der Beschäftigungsförderung verbunden (vgl. Mohr 2009), die sich nicht zuletzt aus einer „skeptischen KostenNutzen-Einschätzung" (Wagner 2007, S. 321) insbesondere der Beschäftigung schaffenden Maßnahmen speiste. We- sentliche Grundannahme des Aktivierungsparadigmas ist, dass Erwerbslosigkeit in erster Linie auf mangelnde Beschäftigungsfähigkeit und/oder unzureichende Arbeitsund Konzessionsbereitschaft der Arbeitsuchenden zurückzuführen sei und damit weniger auf das strukturell zu lösende Problem fehlender Arbeitsplätze und entsprechender Unterbeschäftigung. Im Zuge dieses arbeitsmarktpolitischen Perspektivwechsels wurden Marktersatz und Strukturwirksamkeit zunehmend durch Zielgruppeninklusion, Arbeitsmarktintegration und Beschäftigungsfähigkeit als wesentliche Zielgrößen des Einsatzes geförderter Beschäftigung ersetzt (vgl. Matysik et al. 2011, S. 13). Entsprechend wurden die Förderzahlen - zumindest bis 2004 - deutlich gesenkt und erhebliche Einschränkungen bei den Förderkonditionen vorgenommen. Dies zeigte sich etwa im Bereich der ABM darin, dass deren Laufzeiten reduziert und die bisherige Orientierung des Entgeltniveaus an tariflichen oder ortsüblichen Bestimmungen ebenso gestrichen wurde wie der Beitrag zur Arbeitslosenversicherung (vgl. Wagner 2007, S. 321).

Durch Zusammenlegung der früheren Arbeitslosenhilfe mit der Sozialhilfe im 2005 eingeführten SGB II schließlich fand der „Strukturwandel“ (ebd., S. 322) der Beschäftigungsförderung seinen (vorläufigen) Abschluss durch die gänzliche Abschaffung der Förderung von ABM (2009 im SGB II, 2012 im SGB III). So dominiert nun mit den im Bereich der Grundsicherung geschaffenen AGH ein Förderinstrument, das nicht mehr - wie die früheren ABM als Arbeitsverhältnis, sondern als kurztaktige sozialrechtliche Maßnahme konzipiert ist (vgl. Oschmiansky/Ebach 2009, S. 18). AGH zielen vorrangig auf die kurzfristige Überwindung von qualifikatorischen wie motivationalen Defiziten Arbeitsloser, die nach Einschätzung der Arbeitsverwaltung einer (Wieder-)Eingliederung in den ersten Arbeitsmarkt entgegenstehen (vgl. Hirseland et al. 2010, S. 72f.). Damit wird der Einsatz öffentlich geförderter Beschäftigung primär am Ziel einer Verbesserung individueller Beschäftigungsfähigkeit und einer Erhöhung von Arbeitsmarktchancen ausgerichtet (vgl. Kupka/Wolff 2009).

Bereits kurz nach Einführung des SGB II entzündete sich an dieser neuen Ausrichtung der Beschäftigungsförderung eine in der politischen wie wissenschaftlichen Fachöffentlichkeit breit und kontrovers geführte Debatte. Kritisiert wurde vor allem die strikte Orientierung an der Integration in den ersten Arbeitsmarkt, was gerade mit Blick auf die keineswegs unerhebliche Zahl jener Leistungsempfänger als unzureichend bewertet wurde, die aufgrund besonderer Einschränkungen kaum Beschäftigungschancen haben und denen folglich die dauerhafte Abhängigkeit von Transferzahlungen droht (vgl. u.a. Buestrich 2008, S. 34ff.). Von unterschiedlichen Seiten wurde daher die Ergänzung des bestehenden Förderinstrumentariums um Maßnahmen gerade für besonders schwer vermittelbare Langzeitarbeitslose gefordert. In der Debatte kursierten zahlreiche Vorschläge, die zum Teil unterschiedliche Schwerpunkte aufwiesen und mal als „dritter Arbeitsmarkt“, teils als 
„ehrlicher zweiter Arbeitsmarkt" (Buntenbach 2006) bezeichnet wurden. Auch seitens der damaligen von CDU/ CSU und SPD gebildeten Koalitionsregierung wurde im Frühjahr 2007 unter dem Titel „Perspektiven für Langzeitarbeitslose mit besonderen Vermittlungshemmnissen“ ein Förderkonzept vorgelegt, das im Rahmen der am Bundesministerium für Arbeit und Soziales angesiedelten „Arbeitsgruppe Arbeitsmarkt" entwickelt und in Gestalt des Beschäftigungszuschusses schließlich rechtlich verankert wurde (vgl. BMAS 2007, S. 16ff.).

\section{Der Beschäftigungszuschuss: Ausgestaltung und Zielsetzung}

Der auch als JobPerspektive bekannte, im Zuge der Ende November 2011 beschlossenen arbeitsmarktpolitischen Instrumentenreform modifizierte Beschäftigungszuschuss ist durch eine eng gefasste Zielgruppendefinition gekennzeichnet und soll ausschließlich jenen Langzeitarbeitslosen Beschäftigungsperspektiven eröffnen, die aufgrund sog. Vermittlungshemmnisse besonders schlechte Arbeitsmarktchancen haben. Den fachlichen Hinweisen der Bundesagentur für Arbeit (BA) zufolge zählen dazu u.a. ein fehlender Berufsabschluss oder gesundheitliche Einschränkungen (BA 2010, S. 12). Zudem ist die Förderfähigkeit daran geknüpft, dass derartige Vermittlungshemmnisse die Erwerbsmöglichkeiten der jeweiligen Person nachhaltig beeinträchtigen und einer Eingliederung in den ersten Arbeitsmarkt tatsächlich entgegenstehen (vgl. § 16e Abs. 1 SGB II). Aus diesem Grund ist der eigentlichen Förderphase eine sechsmonatige sog. Aktivierungsphase vorgeschaltet, während der potenzielle Förderkandidaten unter Rückgriff auf Regelinstrumente des SGB II gezielt aktiviert und verstärkte Vermittlungsversuche unternommen werden sollen (vgl. BA 2010). Erst wenn eine Eingliederung in nicht geförderte Beschäftigung weder unmittelbar erfolgen kann noch - der Prognose der betreuenden Vermittlungsfachkraft zufolge - innerhalb der nächsten 24 Monate erwartbar ist, kommt eine Förderung nach $₫ 16 \mathrm{e}$ SGB II in Betracht (zur Implementationspraxis vgl. Bauer et al. 2010).

Der Beschäftigungszuschuss ist als Zuschuss von bis zu $75 \%$ der Bruttolohnkosten konzipiert, den Arbeitgeber zunächst nur gemeinnützige, ab April 2008 auch privatwirtschaftliche - bei Einstellung eines förderfähigen Leistungsberechtigten erhalten können. Mit der Lohnsubvention sollen die Produktivitätseinschränkungen der Zielpersonen finanziell kompensiert und ihre Beschäftigung für Betriebe rentabel gemacht werden (vgl. ebd., S. 274). Nach einer eher schleppenden Anlaufphase stieg der Bestand an bezuschussten Beschäftigungsverhältnissen ab April 2008 deutlich an und erreichte im Januar 2010 mit rund 42.000 den bisherigen Höchststand. Die zugewiesenen Förderfälle gingen von monatlich durchschnittlich knapp 2.500 zwischen
April 2008 und Januar 2010 auf nur noch rund 200 im Juli 2011 zurück (Abbildung 1). Insgesamt wurde mit rund 62.000 Geförderten (BA 2011a) die ursprünglich anvisierte Zielgröße von insgesamt 100.000 Personen (vgl. Deutscher Bundestag 2007, S. 6) nicht erreicht.

Der Beschäftigungszuschuss stellt in mehrfacher Hinsicht eine Korrektur der Aktivierungslogik des SGB II dar. Den Ausgangsüberlegungen zur Begründung der Notwendigkeit eines solchen Instruments zufolge basieren die geringen Beschäftigungschancen der Zielgruppe nicht - wie im Aktivierungsansatz unterstellt - auf mangelnder Arbeitsund Konzessionsbereitschaft, ${ }^{\boldsymbol{B}}$ sondern auf faktischen Einschränkungen ihrer Leistungsfähigkeit. Daher erscheinen aktivierende Maßnahmen, die mit einer Kombination aus finanziellen Anreizen und Sanktionen auf verstärkte Eigeninitiative bei der Arbeitssuche abzielen, als „,unsinnig“ (Bauer et al. 2010, S. 274). Auch steht bei der Zielsetzung nicht mehr die Integration in den ersten Arbeitsmarkt im Vordergrund. Die geförderte Erwerbsintegration soll vielmehr dazu beitragen „Perspektiven zur gesellschaftlichen Teilhabe“ (Deutscher Bundestag 2007, S. 1) zu eröffnen.

Was damit konkret gemeint und beabsichtigt ist, haben die beiden Unterhändler der damaligen Regierungsparteien - der ehemalige nordrhein-westfälische Arbeitsminister KarlJosef Laumann (CDU) und der damalige arbeitsmarktpolitische Sprecher der SPD-Bundestagsfraktion Klaus Brandner - in einem Strategiepapier beschrieben: Während Erwerbslosigkeit eine wesentliche Ursache sozialer Ausgrenzung darstellt, gilt Erwerbsarbeit hingegen als „Schlüssel zur gesellschaftlichen Teilhabe“ (Brandner 2007, S. 6). Erwerbsarbeit gewähre die Mittel einer eigenständigen Unterhaltssicherung als „Voraussetzung für ein selbstbestimmtes Leben“ (ebd.), sei mit "gesellschaftlicher Anerkennung" (ebd.) verbunden und geeignet, „dem Menschen Ordnung, Halt und Stabilität“ (ebd.) zu verleihen. Vor diesem Hintergrund kommt geförderter Beschäftigung die Aufgabe zu, auch jenen auf dem ersten Arbeitsmarkt chancenlosen Arbeitslosen die skizzierten Integrations- und Teilhabeeffekte regulärer Beschäftigung zu erschließen und so die mit Arbeitslosigkeit verbundenen Ausgrenzungsrisiken zu überwinden.

Um dieser Zielstellung gerecht zu werden, wurden die Rahmenbedingungen der geförderten Beschäftigung am Referenzmodell des „Normalarbeitsverhältnisses“ (Mückenberger 1985) ausgerichtet und unterscheiden sich damit wesentlich von denen der AGH des SGB II. So wurden bis Ende 2011 sowohl bei gemeinnützigen wie privatwirtschaftlichen Arbeitgebern Arbeitsverhältnisse gefördert, die - mit Ausnahme des Beitrags zu Arbeitslosenversicherung - sozialversicherungspflichtig sind, in Vollzeit ausgeübt werden, eine tarifliche bzw. ortsübliche Entlohnung

3 Dies scheint übrigens für ALG-II-Empfänger allgemein zu gelten, die in Befragungen in Relation zur Gesamtbevölkerung "eine hohe Arbeitsmotivation und Konzessionsbereitschaft" (Beste et al. 2010, S. 1) aufweisen. 
vorsehen und zumindest Alleinstehenden ein Leben jenseits des Hilfebezugs ermöglichen sollen (vgl. Brandner 2007, S. 10). Zudem bestand erstmals in der deutschen Arbeitsmarkt- und Sozialpolitik die Möglichkeit, die Lohnförderung im Anschluss an die zunächst zweijährige Befristung auch unbefristet fortzusetzen, sofern eine Vermittlung des Geförderten in den ersten Arbeitsmarkt weiterhin ausgeschlossen werden konnte. Mit dem Beschäftigungszuschuss ist folglich eine „starke Normalitätsorientierung" (Bauer et al. 2010, S. 274) verbunden, die in deutlichem Gegensatz zum „Work-First“-Prinzip des SGB II steht, dessen entsprechend rigide Zumutbarkeitsbestimmungen ( $\$ 10$ SGB II) atypische Beschäftigungsformen keineswegs ausschließen.

\section{Teilhabe in der Arbeitsgesellschaft}

Ein Instrument wie der Beschäftigungszuschuss folgt damit einer Perspektive der deutschen wie der europäischen $\mathrm{Ar}$ beitsmarkt- und Sozialpolitik, die Reintegration in den Arbeitsmarkt als Verbesserung sozialer Teilhabe begreift (vgl. Bernhard 2011; Mohr 2007). Aus dem französischen Debattenraum stammend, haben der Begriff soziale Teilhabe und sein Gegenbegriff soziale Ausgrenzung in den vergangenen Jahren an sozialwissenschaftlicher Bedeutung gewonnen. Als zeitdiagnostische Kategorien thematisieren sie jene „simultane Krise der Arbeitsgesellschaft wie ihrer sozialstaatlichen Absicherung" (Ludwig-Mayerhofer 2009, S. 6), die sich bereits gegen Ende der 1970er Jahre andeutete und die in der Zunahme atypischer Erwerbsformen, der Verstetigung von Langzeiterwerbslosigkeit, aber auch im Umbau sozialstaatlicher Sicherungssysteme ihren Ausdruck findet.

Im Anschluss an die Arbeiten von Castel (2000) und Kronauer (2002) fungiert das Begriffspaar zugleich als analytisches Konzept, mit dem die Lebensbedingungen von prekär Beschäftigten, Langzeiterwerbslosen und anderen in den Blick genommen werden, die in besonderem Maße von Ausgrenzungsrisiken betroffen sind. Über kontroverse theoretische Positionen hinweg besteht Einigkeit darin, dass soziale Teilhabe und soziale Exklusion (Ausgrenzung) gleichermaßen relationale wie multidimensionale Konzepte sind. So bemisst sich soziale Teilhabe an den verfügbaren Chancen und Handlungsmöglichkeiten, individuell gewünschte und gesellschaftlich übliche Lebensweisen und -standards realisieren zu können (vgl. Bartelheimer 2004, S. 53). Ausgrenzungsrisiken entstehen dann, wenn die notwendigen, etwa ökonomischen Voraussetzungen zur Realisierung jener Lebensweisen nicht bzw. nur eingeschränkt verfügbar sind. Den zentralen Bezugspunkt stellt dabei die Unterscheidung von zwei zentralen Modi und mehreren Dimensionen sozialer Integration dar. So differenziert Kronauer (2002, S. 151ff.) zwischen der Einbindung in die vielfach erwerbsförmig organisierte gesellschaftliche Arbeits-
ABB.

\section{Zugänge in die Förderphase des Beschäftigungszuschusses}

Absolute Zahlen (Personen)

- Deutschland

Westdeutschland

- Ostdeutschland

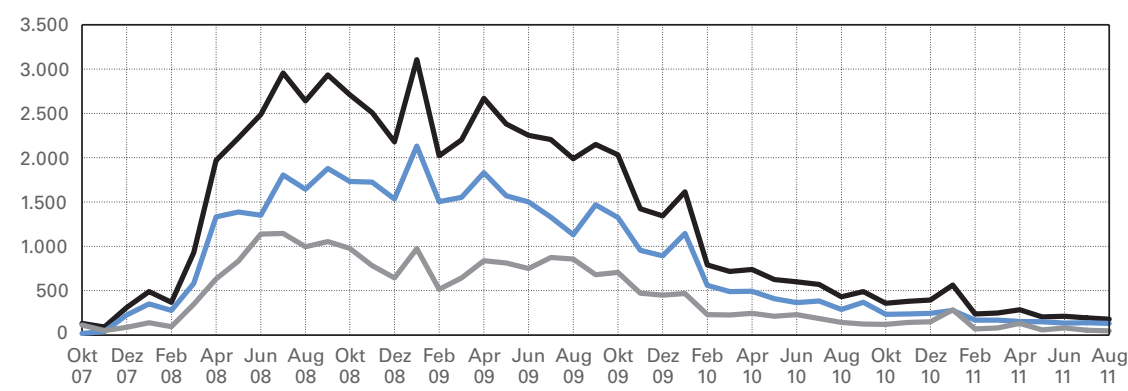

$\begin{array}{llllllllllllllllllllllllll}07 & 07 & 08 & 08 & 08 & 08 & 08 & 08 & 09 & 09 & 09 & 09 & 09 & 09 & 10 & 10 & 10 & 10 & 10 & 10 & 11 & 11 & 11 & 11\end{array}$

Quelle: Statistik der BA 2011a, 2011b.

teilung und soziale Nahbeziehungen (Interdependenz) sowie den materiellen, politisch-institutionellen und kulturellen Teilhabemöglichkeiten an gesellschaftlich anerkannten Lebenschancen und -standards (Partizipation).

Die herausgehobene Bedeutung von Erwerbsintegration bzw. Erwerbsarbeit als zentraler Vergesellschaftungsinstanz moderner (Arbeits-)Gesellschaften, vor allem in Form des „Normalarbeitsverhältnisses“ (Mückenberger 1985), beruht darauf, dass sie den Arbeitenden den sozial anerkannten „Status des Lohnabhängigen“ (Castel 2000, S. 296) zuweist. Dieser umfasst eine eigenständige, gegen Risiken abgesicherte Unterhaltssicherung ebenso wie die über den Lohn ermöglichte Teilhabe an der Konsumkultur. Zudem vermittelt Erwerbsarbeit die Erfahrung kollektiver wie institutioneller sozialer Anerkennung im unmittelbaren Arbeitszusammenhang und darüber hinaus (vgl. Promberger 2008, S. 10). ${ }^{\oplus}$ Im Umkehrschluss werden Teilhabedefizite insbesondere als Folge von (länger andauerndem) Erwerbsausschluss verstanden. So konnte die sozialwissenschaftliche Arbeitslosigkeitsforschung wiederholt zeigen, dass gerade verfestigte Erwerbslosigkeit vielfach materielle und soziale Deprivation nach sich zieht. Erwerbslosigkeit entlässt die von ihr Betroffenen aber auch „aus der durch die Arbeit generierten raumzeitlichen Strukturierung des Alltags in die Strukturlosigkeit“" (ebd., S. 12) und begünstigt gesundheitliche Probleme (klassisch: Jahoda et al. 1975; aktuell: u.a. Kieselbach/Beelmann 2006). Aus diesen Gründen

4 Dies gilt keineswegs für alle Arten von Beschäftigung gleichermaßen. Insbesondere die im Anwachsen begriffenen prekären Beschäftigungsformen weisen vielfach deutliche Einschränkungen bezüglich ihrer Inklusionseffekte auf (vgl. etwa Bartelheimer 2011; Gundert/Hohendanner 2011; Rademacher/Ramos Lobato 2008). 
liegt es nahe, „Arbeitslosigkeit mit Arbeit“ (Promberger 2008, S. 13) zu begegnen, wenn nötig auch mit geförderter Erwerbsarbeit, um auf diese Weise den Wegfall der vergesellschaftenden Wirkung (regulärer) Beschäftigung zumindest zeitweise zu kompensieren.

Entsprechend soll der Beschäftigungszuschuss schwer vermittelbaren Personen im SGB II qua öffentlich bezuschusstem Arbeitslohn „wirtschaftliche Selbstständigkeit“ sowie ein „selbstbestimmtes Leben“ (Brandner 2007, S. 6) ermöglichen und in der Folge ihre Teilhabechancen nachhaltig verbessern. Aus Sicht der politischen Initiatoren stellt die eigenständige Unterhaltssicherung durch Erwerbsarbeit daher die maßgebliche Differenz gegenüber der mit Langzeiterwerbslosigkeit im Regelfall verbundenen finanziellen Abhängigkeit von Grundsicherungsleistungen dar. Mit dieser (normativen) Zielsetzung gewinnt die Perspektive der Geförderten an Bedeutung. Die Erfahrung von Selbstbestimmtheit und Teilhabe, ihr Ge- oder Misslingen sind immer Resultat eines Herstellungsprozesses, der auch beeinflusst wird von subjektiven Lebensumständen, Handlungs-, Wahrnehmungs-, Erfahrungs- und Erlebensmustern. Inwieweit geförderte Beschäftigung zu einer Verbesserung sozialer Teilhabe beiträgt, lässt sich folglich kaum beantworten, ohne den Blick auf die Geförderten und die subjektive Bedeutung zu richten, die sie dieser Form der Erwerbsintegration, ihren Rahmenbedingungen und Folgen zuweisen.

\section{Soziale Teilhabe durch geförderte Beschäftigung?}

In einer qualitativen Studie mit geförderten Langzeitarbeitslosen wurde exploriert, ob und wie mit dem Beschäftigungszuschuss geförderte Arbeit Teilhabeerfahrungen verändert. ${ }^{\boldsymbol{\Theta}}$ Eine erste (praktische) Schwelle bildeten die Integrationsprozesse der Geförderten in den jeweiligen betrieblichen Arbeitszusammenhang. Auch bewirkten die mit dem Eintritt in den Beschäftigungszuschuss gegenüber dem vorangegangenen Grundsicherungsbezug erweiterten ma- teriellen Spielräume im Alltag der Befragten verbesserte Teilhabemöglichkeiten. ${ }^{\bullet}$ Die Fallauswertung zeigte aber die herausgehobene Bedeutung der erlebten Veränderung des sozialen Status für die Teilhabeerfahrungen bzw. die wahrgenommene „Normalisierung“ der Lebensumstände. Aus Platzgründen und wegen dieser - in allen untersuchten Fällen beobachtbaren - Schlüsselfunktion der Statuswahrnehmung für das (gesteigerte) Inklusionsempfinden der Befragten konzentrieren wir uns im Folgenden auf diesen Aspekt.

\subsection{Zugewinn an sozialer Freiheit...}

„Und ich bin ja bloß froh, dass ich von der ARGE weg bin.“ Dieses Zitat eines Befragten zeigt: Der Übergang in die geförderte Beschäftigung wird allgemein als Zugewinn an sozialer Freiheit wahrgenommen. Während des vorangegangenen Langzeitbezugs von ALG II war die Lebensgestaltung der Befragten in hohem Maße durch die Aktivierungslogik des „Förderns und Forderns“ geprägt. Häufig wurde dies als Fremdbestimmung erfahren - nicht zuletzt auch wegen der Unberechenbarkeit, Heterogenität und relativ kurzen Dauer der zugewiesenen Aktivierungs- und Fördermaßnahmen. Dadurch wurde eine auf Kontinuität gestellte Alltags- und Lebensplanung erschwert, mussten die Betroffenen doch mehr oder weniger lange Phasen des Wartens auf das nächste kurzzeitige Aktivierungs- bzw. Beschäftigungsangebot überbrücken. So wird der Eintritt in geförderte Beschäftigung als eine Art Ausbruch aus dem in vielerlei Hinsicht als sinnentleert und entwürdigend empfundenen Alltag eines Hilfeempfängers erfahren: „Das ist was ganz anderes. Also wenn ich weiß, ich bin dafür morgens aufgestanden und konnte auch was machen und bekomme dieses Geld, das ist ein ganz anderes Gefühl, als wenn Sie zu Hause sitzen und andere Leute denken wahrscheinlich von Ihnen noch, Sie sind so ein Schmarotzer, der zu Hause sitzt, weil er gar nichts machen will. Und zur Strafe dafür, dass die Leute so über einen denken, bekommt man dann auch noch kaum Geld von denen zum Leben. Das ist was ganz anderes [für sein Geld zu arbeiten; d. Verf.], kann man überhaupt nicht miteinander vergleichen." (B0111-WM)
5 Hierbei handelt es sich um eine Teilgruppe $(n=20)$ des bundesweit insgesamt rund 150 Personen umfassenden Samples der qualitativen Panelstudie "Armutsdynamik und Arbeitsmarkt. Entstehung, Verfestigung und Überwindung von Hilfebedürftigkeit bei Erwerbsfähigen“. Für detaillierte Angaben zum methodischen Design der Untersuchung vgl. Hirseland/Ramos Lobato 2010. Die Befragung der geförderten Personen wurde erstmalig im Rahmen der dritten von insgesamt vier Erhebungswellen durchgeführt und im Kontext der Evaluation der Beschäftigungsförderung nach § 16 e SGB II ausgewertet. Zentrale Themen der Interviews waren neben der bisherigen Erwerbsbiografie und den Erfahrungen von Erwerbslosigkeit und Leistungsbezug auch die betriebliche Integration im Rahmen der geförderten Beschäftigung, das soziale Umfeld und die Zukunftspläne.
6 Ausführlicher zu beiden Aspekten vgl. Deutscher Bundestag 2011, S. 123ff. Die Förderung betrug durchgängig $75 \%$, das monatliche Nettoentgelt lag zwischen 700 und $1200 €$. Zumeist konnte damit der Bezug von Arbeitslosengeld II (ALG II) überwunden werden, teils unter Einbeziehung andererTransferleistungen wie bspw. dem Kinderzuschlag (§ 6a BKGG). In einigen Fällen reichte der Verdienst, v.a. wegen des jeweiligen Zuschnitts der Bedarfsgemeinschaft, nicht aus, um auf ergänzenden Bezug von ALG II verzichten zu können. Jedoch verbesserte sich auch hier aufgrund der nichtanrechenbaren Freibeträge ( $\$ 30$ SGB II) die Einkommenssituation. 
Die geförderte Beschäftigung vermittelt das Gefühl, eine Aufgabe zu haben, gebraucht und anerkannt zu werden, oder anders gewendet: nicht überflüssig zu sein. In diesem Zusammenhang wird auch die gegenüber den sonstigen Maßnahmen des SGB II vergleichsweise längere Bewilligungsdauer - i.d.R. zwei Jahre statt sechs Monate - des Beschäftigungszuschusses bedeutsam. Bei vielen der Befragten erweist sich der erweiterte Zeithorizont als stabilisierende Grundlage für eine autonomere alltägliche Lebensführung. Die Zeitspanne ermöglicht, den aktuellen Beschäftigungsstatus so wahrzunehmen, als ob er „auf Dauer gestellt“ wäre. Dies begünstigt den Aufbau einer auf Routinisierung und Habitualisierung beruhenden Veralltäglichung erwerbsbezogener Handlungsmuster und damit einer geänderten Selbstwahrnehmung. Die Befragten sehen sich selbst nun nicht mehr als randständige, abhängige Hilfeempfänger, sondern als normalinkludierte Mitglieder der Erwerbsgesellschaft.

Zur Entwicklung dieses Selbstverständnisses trägt auch bei, dass sie für die Dauer ihrer geförderten Beschäftigung keinen weiteren Aktivierungsanforderungen der für sie zuständigen SGB-II-Träger unterliegen. So entfallen nun die häufig als Rechtfertigungszwang erlebten regelmäßig anfallenden Termine und Vorsprachen bei den Grundsicherungsträgern ebenso wie die teils gegen den Willen der Betroffenen erfolgenden Zuteilungen zu Maßnahmen, die zu verweigern nur um den Preis einer Sanktionierung möglich gewesen wäre. Entsprechend wird die Freistellung von unmittelbaren administrativen $\mathrm{Zu}$ - und Eingriffen in die Lebensgestaltung auch als gesteigertes Unabhängigkeitsgefühl beschrieben, das Raum gibt für die Entfaltung eines den eigenen Vorstellungen entsprechenden selbstbestimmten Lebens.

\section{2 ... mit eingeschränkter Perspektive}

Dieses Unabhängigkeitsgefühl ist jedoch oft fragil, wenn sich die Befragten bewusst machen, dass es auf befristeten Beschäftigungsverhältnissen beruht und ihnen die während des Untersuchungszeitraums bestehende Möglichkeit der Entfristung nicht bekannt ist. Daher wird vor allem in den gegen Ende der Förderphase geführten Interviews wiederholt die fehlende Einbindung in die Arbeitslosenversicherung bedeutsam, auch in Form der irrigen Hoffnung, nach Auslaufen der Maßnahme wenigstens einen "normalen“ Arbeitslosenstatus erhalten zu können. Entsprechend wird das antizipierte Ende des geförderten Beschäftigungsverhältnisses als doppelt bedrohlich wahrgenommen: Die Sorge vor erneuter Erwerbslosigkeit und dem damit einhergehenden Verlust an Lebenssicherheit verstärkt sich durch die Sorge vor einer erneuten Abhängigkeit vom administrativen Zugriff des SGB-II-Trägers und einem Rückfall in den als stigmatisierend erlebten gesellschaftlichen Status des Hartz-IV-Empfängers.

Vor diesem Hintergrund ist es nicht verwunderlich, wenn in Fällen, die auch während der geförderten Beschäftigung weiterhin auf ergänzende Grundsicherungsleistun- gen angewiesen sind, das Erleben von Autonomie und Selbstbestimmtheit oftmals trotz des Bezugs von Arbeitslohn eingeschränkt ist bzw. bleibt. Anstatt nun „richtig raus aus Hartz IV“ zu sein - wie dies ein Befragter ausdrückt, der seinen geförderten Lohn mit ALG II aufstocken muss, - wird das fortgesetzte Angewiesensein auf Grundsicherungsleistungen gerade wegen der (geförderten) Erwerbstätigkeit als besonders diskriminierend empfunden: „Ich muss aufstocken, sogar mit Hartz IV, finde ich persönlich eigentlich nicht gut. Wenn Arbeit, wenn eine Vollzeitarbeit, dann auch richtig. Wie gesagt, kein Hartz IV. Also das muss schon fair bezahlt werden, sollte fair bezahlt werden. [...] Na, man will ja weg von Hartz IV. Trotz Vollzeitarbeit, 40-Stunden-Woche, das ist eigentlich nicht normal, finde ich. Sollte auch nicht normal werden. [...] Also ich muss trotzdem alles ausfüllen. Trotz dass ich arbeiten gehe, ich muss komplett alles ausfüllen, Hartz-IV-Bescheid, alles, und mit Einkommensbescheiden kommen, so wie ein Hartz-IV-Empfänger auch. Das ist eben nicht so schön, finde ich. Ja, das zieht einen auch ein bisschen runter dann." (C0076-OG)

Die innerhalb der geförderten Beschäftigung geleistete Arbeit wird als „,vollwertige“ Arbeit empfunden, die daher - wie in der Forderung nach „fairer“ Bezahlung ausgedrückt - ein Leben zumindest jenseits der Bedürftigkeitsgrenze ermöglichen sollte. In dieser Erwartung verknüpft sich die materielle Frage mit der nach sozialer Anerkennung und Gerechtigkeit. Indem Arbeitende und Nicht-Arbeitende seitens der Grundsicherungsträger den gleichen administrativen Prozeduren unterzogen werden, wird aus Sicht des Befragten Ungleiches gleich behandelt, was gegen die sonst gesellschaftlich legitimierte Regel, Gleiches gleich zu behandeln, verstößt. „Trotz Vollzeitarbeit“ nicht nur auf staatliche Unterstützung angewiesen zu sein, sondern zudem den gleichen behördlichen Prozeduren unterworfen zu werden wie ein mittelloser Erwerbsloser, wird als Kränkung wahrgenommen. Die Bedürftigkeitsprüfung wird subjektiv als Diskriminierung erfahren, als Verweigerung der Anerkennung eines aus dem Erwerbsstatus abgeleiteten Anspruchs auf ein selbstbestimmtes, nicht vom empfundenen Wohlwollen Dritter abhängiges und reglementiertes Leben. Was aus Sicht des Befragten mit Blick auf erwerbslose Leistungsbezieher als noch (halbwegs) legitim erscheinen mag, wird für ihn vor dem Hintergrund seines als solchen wahrgenommenen Arbeitnehmerstatus kritisierbar. Aufgrund seiner Arbeit sieht er sich in einer sozialen Position, die nach einer Form symbolischer Anerkennung verlangt, die nicht zuletzt auch in prozeduralen Fragen seiner behördlichen „Fallbehandlung“ Ausdruck finden soll.

\subsection{Verbessertes Selbstwertgefühl}

Demzufolge erweist sich das über den Beschäftigungszuschuss verdiente Geld bezüglich der Frage der Selbstbestimmtheit und des Autonomieerlebens nicht nur in seiner quantitativen Dimension als bedeutsam, sondern 
auch in seiner symbolischen Funktion als Statusmarker. Das ist zunächst keineswegs selbstverständlich, stellt der über den Beschäftigungszuschuss bezogene Lohn doch weniger ein nach Produktivitäts- und Marktkriterien vergütetes Einkommen dar, sondern überwiegend eine nach wie vor vom Grundsicherungsträger gewährte Sozialleistung. Schließlich soll mit der Förderung der Bezug von Grundsicherungsleistungen substituiert und eine in der Person der Geförderten liegende Minderung der Leistungs- und Beschäftigungsfähigkeit kompensiert werden. Dennoch besteht für die Befragten der wesentliche, symbolisch bedeutsame Unterschied zum Bezug von Grundsicherungsleistungen in der Form der Leistungsgewährung: Nicht nur entfällt nun die regelmäßig anfallende Bedürftigkeitsprüfung, sondern, fast wichtiger noch, der Beschäftigungszuschuss erreicht die Betroffenen in Form einer an die individuelle Leistungserbringung gekoppelten Zahlung. Indem das geförderte Arbeitsentgelt vom Arbeitgeber ausbezahlt wird, unterscheidet es sich aus Sicht der Befragten nicht von einem normalen Erwerbseinkommen.

Aufgrund dieser formalen Unterschiede zu den Grundsicherungsleistungen wird der bezuschusste Lohn selbst zum Medium einer Renormalisierung des individuell empfundenen gesellschaftlichen Status. Die Geförderten sehen ihre gesellschaftliche Position nun als kaum mehr von der anderer Arbeitnehmer unterscheidbar, da sie die bezogene Vergütung als „selbstverdientes“, durch eigene Leistung erworbenes Geld betrachten können. Dies ermöglicht ihnen die Übernahme einer Selbstachtung und Selbstsicherheit fördernden Verdienerperspektive. Wird diese zur Basis des eigenen Selbstverständnisses, gelingt es sogar, den fortgesetzten Bezug von ergänzendem ALG II als weniger stigmatisierend zu empfinden. Dies geschieht durch Umdeutungen der aufstockenden Grundsicherung zur "Ausgleichszahlung“ - etwa für weiterhin zu leistende Kinderbetreuung im Falle einer allein erziehenden Mutter - oder von deren Beantragung als bloße Formsache: „Da gebe ich einfach nur noch alle halbe Jahre meinen Antrag ab“.

Auch zeigt sich das neu bzw. wieder erlangte Selbstbewusstsein in der Art, wie die Bewältigung der mit einer selbstbestimmten Lebensführung einhergehenden alltäglichen Handlungsanforderungen erlebt wird. Zwar berichten einige der Befragten, sie hätten zunächst Schwierigkeiten gehabt, den mit dem Wechsel in geförderte Beschäftigung einhergehenden Anforderungen etwa einer eigenständigen Regelung ihrer finanziellen Verpflichtungen - wie Fahrtkosten, Rundfunkgebühren oder Unterhaltszahlungen nachzukommen. Derart gestiegene Ansprüche an die Fähigkeit zu planerischem „Finanzmanagement" wirkten zunächst belastend. Dennoch überwiegt schließlich die Erfahrung einer als Autonomiegewinn erlebten Erweiterung von Entscheidungsspielräumen und -optionen. So wird in einigen der Interviews mit spürbarer Emphase davon berichtet, Eigenverantwortung auch in Fragen der all- täglichen Lebensbewältigung übernehmen zu können: „Ja und jetzt BEZ [Beschäftigungszuschuss; d. Verf.], jetzt freue ich mich total, wie gesagt, dass ich mein eigenes Geld verdiene, Verantwortung darüber habe, die Miete zu zahlen und... genau, also Eigenverantwortung zu übernehmen, genau." (B0090-WG)

Das Gefühl, sein Leben selbst in die Hand nehmen zu können, wirkt sich auch auf das Konsumverhalten aus. In einer Konsumgesellschaft wird die Teilhabe an dem vielfältigen Güterangebot und die damit verbundene Möglichkeit, sich selbst durch eigene Wahlen in seiner Individualität $\mathrm{zu}$ bestätigen, zu einem wichtigen Moment der Erfahrung sozialer Teilhabe. Während, wie in den Interviews mehrfach zum Ausdruck gebracht wird, die Grundsicherungsleistungen „nur zum Überleben“ gereicht hätten und zum Teil sogar die Hilfe karitativer Einrichtungen wie der Tafeln in Anspruch genommen werden musste, verbessert und stabilisiert sich nun die materielle Versorgungslage. Die finanziellen Gestaltungsspielräume erweitern sich, vor allem in Bezug auf Ernährung, Kleidung und Freizeitgestaltung.

So berichten die Befragten, dass sie sich öfter qualitativ höherwertige Lebensmittel leisten können und nicht mehr ausschließlich auf als Billigartikel bezeichnete Discounterwaren angewiesen sind. Derartige kleine Freiheiten tragen maßgeblich zu dem Gefühl sozialer Aufwertung bei, von dem die Befragten berichten. Von besonderer Bedeutung hierbei sind auch erweiterte Spielräume für soziale und Freizeitaktivitäten, die während des Grundsicherungsbezugs zwangsläufig besonders eingeschränkt werden mussten. Diese vorrangig im Bereich der sozialinvestiven Ausgaben vorgenommenen Einsparungen führten vielfach zum Abbau sozialer Teilhabe durch sukzessiven Verlust sozialer Kontakte und zunehmender (Selbst-)Isolation. Viele der Befragten erfahren es daher als Bereicherung ihres Alltagslebens, auch mal wieder eine Gaststätte besuchen, ins Kino oder Schwimmbad gehen zu können, ohne sich dieses vom Munde absparen zu müssen. Über derartige Aktivitäten erschließen sich oft zugleich neue Bekanntschaften. Ebenso werden alte Bekanntschaften, die während des Hilfebezugs zum Erliegen kamen, reaktiviert und auf diesem Wege wird das persönliche soziale Netzwerk, das vielfach nur noch - wenn überhaupt - aus anderen Hilfebeziehern bestand, erweitert.

Auch hier zeigt sich, dass der Zugewinn an alltäglichen Freiheiten nicht den vergrößerten finanziellen Spielräumen allein zu verdanken ist. Vielmehr wird in den Interviews Stolz zum Ausdruck gebracht, diesen bescheidenen Zugewinn an Lebensqualität aus eigener Kraft erreicht zu haben. Denn aus der durch den Beschäftigungszuschuss ermöglichten Verdienerperspektive betrachtet, muss die Verausgabung des Geldes nicht mehr weiter gerechtfertigt werden - sei es vor dem Grundsicherungsträger oder der „fiktiven Geber-Gemeinschaft“ der Steuerzahler. Das, was sich die Befragten leisten können, leisten sie sich nun guten Gewissens und ohne Scham. 


\section{Fazit: Teilhabechancen und -restrik- tionen geförderter Beschäftigung}

Die hier vorgestellten Befunde und Beobachtungen verweisen darauf, dass sich die Teilhabechancen von Personen, die unter den gegenwärtigen Arbeitsmarktbedingungen ein hohes Risiko dauerhafter Ausgrenzung von Erwerbsteilhabe tragen, durch den Beschäftigungszuschuss erweitern lassen. Das dürfte zumindest auf jenen in unserer Untersuchung angetroffenen Typ des Hilfe beziehenden Langzeitarbeitslosen zutreffen, dessen normativer Orientierungsrahmen trotz länger bestehender Vermittlungshemmnisse keineswegs erwerbsfern, sondern nach wie vor am Modell einer erwerbsarbeitszentrierten Lebensführung und einem korrespondierenden Arbeitsethos ausgerichtet ist. Den Hintergrund des gesteigerten Teilhabeerlebens bildet zweifelsohne der Umstand, dass die Befragten ihre lang andauernden Hilfebezugsphasen durchgehend als deprivierend erfahren haben. Das betrifft nicht nur die Hinnahme materieller Einschränkungen. Gravierender erscheint der Verlust an Kontrolle über das eigene Leben, das weitgehend durch die Vorgaben und Aktivierungsrhythmen der Grundsicherungsträger bestimmt wurde. Auch wurde die Tatsache, vom Transferleistungsbezug als gegenleistungsfrei bezogenem Einkommen abhängig zu sein, weitgehend als Verletzung des Selbstwertgefühls wahrgenommen.

Vor diesem Hintergrund führte der Beschäftigungszuschuss zu einem gesteigertem Selbstwertgefühl, einem Zugewinn an erlebter Selbstbestimmung und Teilhabe. Selbstbestimmung setzt das Vorhandensein von selbst wählbaren und praktikablen Handlungsalternativen voraus. Im Falle des Beschäftigungszuschusses ergeben sich diese sowohl aufgrund des geänderten institutionellen wie auch empfundenen sozialen Status der Geförderten sowie der - über die Befriedigung des rein Lebensnotwendigen hinaus - erweiterten finanziellen Spielräume. In unserer Untersuchung traten freilich auch deutliche Hinweise darauf auf, dass diese Effekte geförderter Beschäftigung unterschiedlich deutlich ausgeprägt sein können. Einschränkungen ergaben sich insbesondere bei fortbestehender Angewiesenheit auf ergänzende Grundsicherungsleistungen, vor allem aber bei ungewissen Anschlussperspektiven gegen Ende der Förderphase und drohendem Verlust der erreichten Teilhabezuwächse. Was die Stabilität der Inklusions- und Teilhabeeffekte betrifft, befinden sich die Betroffenen also in einer höchst vulnerablen, von den wirtschaftlichen und/oder politischen Entscheidungen anderer abhängigen Lage. Dies betrifft insbesondere diejenigen, bei denen die geförderte Beschäftigung nicht zu einer Steigerung ihrer Beschäftigungsfähigkeit nach den Kriterien des ersten Arbeitsmarkts führt und bei denen persönliche Einschränkungen als verfestigte Vermittlungshemmnisse die Erwerbsintegration nachhaltig be- bzw. verhindern.

Von der Frage einer nachhaltigen Teilhabesicherung abgesehen, zeigen die Untersuchungsergebnisse, dass sich durch den Eintritt in geförderte Beschäftigung Exklusions- risiken subjektiv wie objektiv verringern lassen und Zugewinne an Teilhabe ermöglicht werden. Von besonderer Bedeutung hierfür sind erstens die Veränderung des institutionellen Status und die daran anknüpfende (relative) Unabhängigkeit nicht nur von Transferleistungen, sondern auch von den Aktivierungsanforderungen der Grundsicherungsträger; zweitens, dass im Beschäftigungszuschuss das Geld zum Lebensunterhalt in erfahrbarem Zusammenhang mit selbst erbrachten Arbeitsleistungen steht. Das bezogene Geld kann so als „eigenes“, selbstverdientes Geld begriffen und ein darauf gegründetes Selbstbewusstsein als Basis einer empfunden Statusaufwertung und eines gesteigerten Inklusionsempfindens entwickelt werden. Nicht zuletzt ist hierfür drittens die - verglichen mit sonstigen Maßnahmen des SGB II - längere Laufzeit der Förderung bedeutsam. Diese bietet den erforderlichen zeitlichen Rahmen, in dem ein eigenes, als selbstbestimmt erlebtes Leben durch die Wiederaneignung und Stabilisierung normalisierter Erwartungs- und Lebensführungsmuster zumindest ansatzweise erfahrbar wird. Eine nachhaltige Teilhabesicherung bedarf daher einer mehr als nur kurzfristigen Erwerbsinklusion.

Es wird zu beobachten bleiben, inwieweit die mit der Instrumentenreform einhergehenden Änderungen des Beschäftigungszuschusses die Chancen der angesprochenen Zielgruppe von Langzeitarbeitslosen auf (nachhaltige) Teilhabe schmälern. Dabei dürfte sich neben der Frage einer existenzsichernden Entlohnung vor allem die Ausgestaltung der Förderdauern und der Ausschluss privatwirtschaftlicher Arbeitgeber als zentral erweisen. Das beschriebene Gefühl sozialer Aufwertung als subjektiver Basis des Teilhabeempfindens ist nach unseren Beobachtungen eng mit der Bewilligungsdauer verknüpft. Hier besteht durch die in der Neuausrichtung vorgenommene Beschränkung der Förderdauern auf max. 24 Monate innerhalb von fünf Jahren und die nach wie vor bestehende budgetäre Verknüpfung der Förderung mit dem Eingliederungstitel der Jobcenter das Risiko einer nicht nur restriktiveren Vergabe, sondern auch der faktischen Verkürzung von Förderdauern. Der Ausschluss privatwirtschaftlicher Arbeitgeber könnte zudem die in einigen Fällen beobachtete Brückenfunktion geförderter Beschäftigung in den ersten Arbeitsmarkt weiter erschweren. Beides könnte, so steht zu befürchten, die in unserer Untersuchung zutage getretenen positiven Teilhabeeffekte unterlaufen. Ähnliches gilt im Bereich der Grundsicherung auch für andere Formen geförderter Beschäftigung, die einerseits, wie die AGH, unterjährig sind und gerade nicht auf dem „Lohnprinzip“ beruhen, oder andererseits, wie etwa der Kommunalkombi oder die Bürgerarbeit, nicht direkt die hier untersuchte Zielgruppe von Langzeitarbeitslosen adressieren und deren Konditionen zudem so ausgerichtet sind, dass der Aktivierungsaspekt trotz Arbeit betont bleibt. Ob es den Betroffenen unter diesen Bedingungen gelingt, geförderte Beschäftigung nachhaltig mit dem für sie symbolisch bedeutsamen Wert der Teilhabe an „richtiger“ Arbeit und den daran geknüpften Folgen in Verbindung zu setzen, bedarf weiterer em- 
pirischer Klärung. Womöglich verweisen die hier vorgestellten Untersuchungsergebnisse auch darauf, dass Aspekten der Teilhabe im Zusammenhang mit der Grundsicherung für Arbeitsuchende insgesamt mehr Aufmerksamkeit geschenkt werden sollte.

\section{LITERATUR}

Bartelheimer, P. (2004):Teilhabe, Gefährdung und Ausgrenzung als Leitbegriffe der Sozialberichterstattung, in: SOFI-Mitteilungen (32), S. 47-61

Bartelheimer, P. (2011): Unsichere Erwerbsbeteiligung und Prekarität, in: WSIMitteilungen 63 (8), S. 386-393, http://www.boeckler.de/wsi_2885.htm

Bauer, F./Franzmann, M./Fuchs, P./Jung, M. (2010): Unbefristet öffentlich geförderte Beschäftigung. Ein Novum in der aktivierenden Arbeitsmarktpolitik. Zwischenergebnisse aus einer Implementationsanalyse zu § 16e SGB II, in:

Sozialer Fortschritt 59 (10-11), S. 273-278

Bellmann, L./Hohendanner, C./Promberger, M. (2006): Welche Arbeitgeber nutzen Ein-Euro-Jobs? Verbreitung und Einsatzkontexte der SGB II-Arbeitsgelegenheiten in deutschen Betrieben, in: Sozialer Fortschritt 55 (8), S. 201-207 Bernhard, S. (2011): Inklusionspolitik als Beratung. Emergenz und Dynamik eines europäischen Politikfeldes, in: Österreichische Zeitschrift für Soziologie 36 (1), S. 28-47

Beste, J./Bethmann, A./Trappmann, M. (2010): Arbeitsmotivation und Konzessionsbereitschaft: ALG-II-Bezug ist nur selten ein Ruhekissen, IAB-Kurzbericht (15), Nürnberg

Brandner, K. (2007): Arbeit für Langzeitarbeitslose ohne Chancen auf dem regulärem Arbeitsmarkt, o.O.

Bude, H./Willisch, A. (Hrsg.) (2006): Das Problem der Exklusion. Ausgegrenzte, Entbehrliche, Überflüssige, Hamburg

Bude, H./Willisch, A. (Hrsg.) (2008): Exklusion. Die Debatte über die „Überflüssigen", Frankfurt a. M.

Buestrich, M. (2008): Ein dritter Arbeitsmarkt - Wozu?, Münster

Buhr, P./Leibfried, S. (2009): Ist die Armutsbevölkerung in Deutschland exkludiert? in: Stichweh, R./Windolf, P. (Hrsg.): Inklusion und Exklusion. Analysen zur Sozialstruktur und sozialen Ungleichheit, Wiesbaden, S. 103-122

Bundesagentur für Arbeit (BA) (2010): SGB II-Arbeitshilfe: Leistungen zur Beschäftigungsförderung nach § 16e SGB II - JobPerspektive, Nürnberg

Bundesagentur für Arbeit (BA) (2011a): Statistik der Bundesagentur für Arbeit. Zeitreihen zu arbeitsmarktpolitischen Instrumenten 2011, Deutschland, Nürnberg

Bundesagentur für Arbeit (BA) (2011 b): Statistik der Bundesagentur für Arbeit. Zeitreihen zu arbeitsmarktpolitischen Instrumenten 2011, West- und Ostdeutschland, Nürnberg

Bundesministerium für Arbeit und Soziales (BMAS) (2007): Bericht der "Arbeitsgruppe Arbeitsmarkt", 0.O

Buntenbach, A. (2006): „Ehrlicher zweiter Arbeitsmarkt" ist notwendig. EinEuro-Jobs durch sozialversicherungspflichtige öffentlich geförderte Beschäftigung ersetzen, in: Soziale Sicherheit 55 (10), S. 233-242

Castel, R. (2000): Die Metamorphosen der sozialen Frage: eine Chronik der Lohnarbeit, Konstanz

Deutscher Bundestag (2007): Entwurf eines Zweiten Gesetzes zur Änderung des Zweiten Buches Sozialgesetzbuch. Verbesserung der Beschäftigungschancen von Menschen mit Vermittlungshemmnissen. Gesetzentwurf der Fraktionen der CDU/CSU und SPD, BT-Dr. 16/5715, Berlin

Deutscher Bundestag (2011): Unterrichtung durch die Bundesregierung. Endbericht der Evaluation der Leistungen zur Beschäftigungsförderung nach $\S 16 e$ Absatz 10 des Zweiten Buches Sozialgesetzbuch, BT-Dr. 17/6880 Gundert, S./Hohendanner, C. (2011): Leiharbeit und befristete Beschäftigung Soziale Teilhabe ist eine Frage von stabilen Jobs, IAB-Kurzbericht (4), Nürnberg

Hirseland, A./Grimm, N./Ritter, T. (2010): Aktivierung zur Arbeit? Zum Gegenstandsbezug qualitativer Forschungsansätze in der Arbeitslosenforschung in Zeiten des SGB II, in: Arbeits- und industriesoziologische Studien (1), S. 71-85 Hirseland, A./Ramos Lobato, P. (2010): Armutsdynamik und Arbeitsmarkt. Entstehung, Verfestigung und Überwindung von Hilfebedürftigkeit bei Erwerbsfähigen, IAB-Forschungsbericht (3), Nürnberg
Jahoda, M./Lazarsfeld, P. F./Zeisel, H. (1975): Die Arbeitslosen von Marienthal. Ein soziographischer Versuch über die Wirkung langandauernder Arbeitslosigkeit, Frankfurt a. M.

Kieselbach, T./Beelmann, G. (2006): Arbeitslosigkeit und Gesundheit. Stand der Forschung, in: Hollederer, A./Brand, H. (Hrsg.): Arbeitslosigkeit, Gesundheit und Krankheit, Bern, S. 13-31

Koch, S./Kupka, P. (2007): Geförderte Beschäftigung für leistungsgeminderte Langzeitarbeitslose? Expertise im Auftrag der Friedrich-Ebert-Stiftung, Bonn Koch, S./Kvasnicka M./Wolff, J. (2010): Beschäftigungszuschuss im SGB II. Ein neues Instrument als Ultima Ratio, IAB-Kurzbericht (2), Nürnberg

Kronauer, M. (2002): Exklusion. Die Gefährdung des Sozialen im hoch entwickelten Kapitalismus, Frankfurt a. M.

Kupka, P./Wolff, J. (2009): Darf's noch etwas mehr sein? Geförderte Beschäftigung in der Wirtschaftskrise. in: Sozialer Fortschritt 58 (11), S. 249-257

Lessenich, S. (2008): Die Neuerfindung des Sozialen. Der Sozialstaat im flexiblen Kapitalismus, Bielefeld

Ludwig-Mayerhofer, W. (2009): Exklusion als soziologisches Konzept, in: Sozialer Sinn 10 (1), S. 3-28

Matysik, A./Rosenthal, P./Sommer, J. (2011): Öffentlich geförderte sozialversicherungspflichtige Beschäftigung in Deutschland. Aktuelle Instrumente, Programme und Konzepte. Expertise im Auftrag der Abteilung Wirtschafts- und Sozialpolitik der Friedrich-Ebert-Stiftung, Bonn

Mohr, K. (2007): Soziale Exklusion im Wohlfahrtsstaat. Arbeitslosenversicherung und Sozialhilfe in Großbritannien und Deutschland, Wiesbaden

Mohr, K. (2009): Von „Welfare zur Workfare“? Der radikale Wandel der deutschen Arbeitsmarktpolitik, in: Bothfeld, S./Sesselmeier, W./Bogedan, C. (Hrsg.) Arbeitsmarktpolitik in der sozialen Marktwirtschaft. Vom Arbeitsförderungsgesetz zum Sozialgesetzbuch II und III, Wiesbaden, S. 49-60

Mückenberger, U. (1985): Die Krise des Normalarbeitsverhältnisses. Hat das Arbeitsrecht noch Zukunft?, in: Zeitschrift für Sozialreform 31 (7), S. 415-434 Oschmiansky, F./Ebach, M. (2009): Aktive Arbeitsmarktpolitik im Wandel, in: Aus Politik und Zeitgeschichte 59 (27), S. 15-20

Promberger, M. (2008): Arbeit, Arbeitslosigkeit und soziale Integration, in: Aus Politik und Zeitgeschichte 58 (40/41), S. 7-15

Rademacher, C./Ramos Lobato, P. (2008): „Teufelskreis oder Glücksspirale?" Ungleiche Bewältigung unsicherer Beschäftigung, in: Eickelpasch, R./Rademacher, C./Ramos Lobato, P. (Hrsg.): Metamorphosen des Kapitalismus - und seiner Kritik, Wiesbaden, S. 118-147

Wagner, A. (2007): Beschäftigungsperspektiven für Langzeiterwerbslose ABM, Arbeitsgelegenheiten und öffentlich finanzierte Beschäftigung, in: WSIMitteilungen 60 (6), S. 320-327, http://www.boeckler.de/cps/rde/xbcr/hbs/ wsimit_2007_06_wagner.pdf

\section{AUTOREN}

ANDREAS HIRSELAND, Dr., Soziologe, ist stellvertretender Leiter des For schungsbereichs „Erwerbslosigkeit und Teilhabe” am Institut für Arbeitsmarkt und Berufsforschung (IAB) in Nürnberg. Arbeitsschwerpunkte: Sozialstaatsforschung, Ungleichheitssoziologie, Qualitative Forschungsmethoden.

andreas.hirseland@iab.de

PHILIPP RAMOS LOBATO, M.A., Soziologe, ist wissenschaftlicher Mitarbeiter der Stabsstelle „Forschungskoordination“ am IAB in Nürnberg. Arbeitsschwerpunkte: Arbeits- und Ungleichheitssoziologie, Qualitative Forschungsmethoden.

philipp.ramos-lobato@iab.de

TOBIAS RITTER, Dipl.-Soz., Soziologe, ist wissenschaftlicher Mitarbeiter am Institut für Sozialwissenschaftliche Forschung e.V. (ISF) München. Arbeitsschwerpunkte: Arbeits- und Techniksoziologie.

tobias.ritter@isf-muenchen.de 